\title{
Promoter alteration causes transcriptional repression of the POMGNT1 gene in limb-girdle muscular dystrophy type 20
}

\begin{abstract}
Madalina Raducu ${ }^{1}$, Jonathan Baets ${ }^{2,3}$, Oihane Fano ${ }^{1}$, Rudy Van Coster ${ }^{4}$ and Jesús Cruces ${ }^{\star, 1}$
Limb-girdle muscular dystrophy type 20 (LGMD20) belongs to a group of rare muscular dystrophies named dystroglycanopathies, which are characterized molecularly by hypoglycosylation of $\alpha$-dystroglycan ( $\alpha$-DG). Here, we describe the first dystroglycanopathy patient carrying an alteration in the promoter region of the POMGNT1 gene (protein 0-mannose $\beta$-1,2-N-acetylglucosaminyltransferase 1 ), which involves a homozygous 9-bp duplication (-83_-75dup). Analysis of the downstream effects of this mutation revealed a decrease in the expression of POMGNT1 mRNA and protein because of negative regulation of the POMGNT1 promoter by the transcription factor ZNF202 (zinc-finger protein 202). By functional analysis of various luciferase constructs, we localized a proximal POMGNT1 promoter and we found a $75 \%$ decrease in luciferase activity in the mutant construct when compared with the wild type. Electrophoretic mobility shift assay (EMSA) revealed binding sites for the Sp1, Ets1 and GATA transcription factors. Surprisingly, the mutation generated an additional ZNF202 binding site and this transcriptional repressor bound strongly to the mutant promoter while failing to recognize the wild-type promoter. Although the genetic causes of dystroglycanopathies are highly variable, they account for only $50 \%$ of the cases described. Our results emphasize the importance of extending the mutational screening outside the gene-coding region in dystroglycanopathy patients of unknown aetiology, because mutations in noncoding regions may be the cause of disease. Our findings also underline the requirement to perform functional studies that may assist the interpretation of the pathogenic potential of promoter alterations.
\end{abstract}

European Journal of Human Genetics (2012) 20, 945-952; doi:10.1038/ejhg.2012.40; published online 14 March 2012

Keywords: dystroglycanopathy; POMGNT1; promoter; limb-girdle muscular dystrophy type 20

\section{INTRODUCTION}

Dystroglycanopathies are a heterogeneous group of congenital muscular dystrophies characterized by hypoglycosylation of $\alpha$-dystroglycan $(\alpha-D G)$, an extensively glycosylated peripheral membrane protein, first isolated from skeletal muscle. ${ }^{1}$ Dystroglycan is the central element of the dystrophin-glycoprotein complex (DGC) and connects the cytoskeleton to components of the extracellular matrix, such as laminin- $\alpha 2$, neurexin, agrin, perlecan and pikachurin. ${ }^{2}$ The correct $O$-glycosylation of $\alpha$-DG is essential to maintain the structural stability of the sarcolemma in the muscle cell. ${ }^{3}$ Synthesis of the O-mannosyl glycan NeuAc $\alpha 2-3 \mathrm{Gal} \beta 1-4 \mathrm{GlcNAc} \beta 1-2 \mathrm{Man} \alpha-\mathrm{S} / \mathrm{T}$, which is essential for dystroglycan to bind normally to laminin, begins with the addition of mannose moieties to serine and threonine residues from the mucin domain of $\alpha$-DG because of the cooperative activity of the glycosyltransferases POMT1 and POMT2 (protein O-mannosyltransferases 1 and 2). ${ }^{4}$ The second step is catalyzed by POMGNT1 (protein O-mannose $\beta$-1,2-N-acetylglucosaminyltransferase 1 ), which transfers $\mathrm{N}$-acetylglucosamine residues to O-linked mannose. ${ }^{5}$ The other enzymes that complete the extension of the tetrasaccharide chain have not yet been identified. However, mutations in the FKTN (fukutin), FKRP (fukutin-related protein) and LARGE (like-glycosyltransferase) genes have been described in patients with defective $\alpha$-DG glycosylation. ${ }^{6}$ POMGNT1 mutations (MIM 606822) were first detected in muscle-eye-brain disease (MEB, MIM 253280), ${ }^{5}$ and they are known to impair the enzymatic activity of this protein. ${ }^{7}$ Subsequently, the clinical spectrum was expanded to include Fukuyama congenital muscular dystrophy (FCMD, MIM 253800), a mild form of Walker-Warburg syndrome (WWS, MIM 236670), and a single patient with limb-girdle muscular dystrophy type $2 \mathrm{O}$ (LGMD2O, MIM 613157). ${ }^{8,9}$ WWS, MEB and FCMD are the most severe forms of these conditions and they have common clinical manifestations, including muscular dystrophy and structural abnormalities of the brain and eye. ${ }^{6}$ Various forms of LGMD without brain and eye involvement are ranked at the milder end of the clinical spectrum. $^{10}$

Here we describe the first dystroglycanopathy patient carrying a mutation in the POMGNT1 promoter region. We present the complete functional analysis of the wild-type POMGNT1 gene promoter, and we define the transcriptional and translational consequences of what is the first mutation in the promoter region of the POMGNT1 gene known to give rise to the LGMD2O phenotype. This mutation promoted the binding of the transcriptional repressor ZNF202 (zincfinger protein 202), provoking the downregulation of POMGNT1 gene expression and, ultimately, defective $\alpha$-DG glycosylation. Our study highlights the importance of functional characterization of promoters in dystroglycanopathies of unknown genetic causes.

${ }^{1}$ Departamento de Bioquímica, Instituto de Investigaciones Biomédicas 'Alberto Sols' UAM-CSIC, Facultad de Medicina, Universidad Autónoma de Madrid, Madrid, Spain; ${ }^{2}$ Neurogenetics Group, VIB Department of Molecular Genetics, Antwerpen, Belgium; ${ }^{3}$ Laboratory of Ultrastructural Neuropathology, Institute Born-Bunge, University of Antwerp, Antwerpen, Belgium; ${ }^{4}$ Department of Pediatrics, Division of Pediatric Neurology and Metabolism, Ghent University Hospital, Ghent, Belgium

*Correspondence: Professor J Cruces, Departamento de Bioquímica, Facultad de Medicina, Universidad Autónoma de Madrid, Calle Arzobispo Morcillo 4, Madrid 28029, Spain. Tel: +34 91497 5406; Fax: +34 91585 4401; E-mail: jesus.cruces@uam.es

Received 25 November 2011; revised 11 January 2012; accepted 2 February 2012; published online 14 March 2012 


\section{MATERIALS AND METHODS}

\section{Patient}

The patient was an 11-year-old Belgian with LGMD that was associated with abnormal $\alpha$-DG glycosylation on histological examination of a muscle biopsy.

\section{Histological analysis and immunohistochemistry}

A biopsy of the quadriceps muscle was performed at the age of 4 years and the sample was analyzed by histological, histochemical, histoenzymatic and immunohistochemical techniques, as described previously. ${ }^{11}$ The antibodies against dystroglycan used were mouse monoclonal $\alpha$-dystroglycan, IIH6C4 (1:500, Millipore, Schwalbach, Germany) and mouse monoclonal anti- $\beta$-dystroglycan (1:200, NCL-b-DG, Novocastra, Newcastle upon Tyne, UK). The avidin-biotin complex technique was used.

\section{Mutation analysis}

Genomic DNA from the patient and his parents was isolated from total blood samples using standard techniques. The coding exons, flanking intronic regions and polyadenylation sites of the POMT1, POMT2, POMGNT1, FKTN, FKRP, $L A R G E$ and DAG1 genes were screened. A $5^{\prime}$-upstream region of $\sim 650$ bp was also screened for each gene. DNA samples taken from 200 healthy individuals were used as controls.

\section{Cloning of the $5^{\prime}$-upstream region of the hPOMGNT1 gene} A 2070-bp 5'-flanking region of the human POMGNT1 gene up to the translation start codon (ATG) was amplified by PCR (Supplementary Table S1). The PCR product was cloned between the HindIII and XhoI sites of the promoterless pXP2 plasmid upstream of the firefly luciferase gene, ${ }^{12}$ generating the construct pXP2(-1426/+644). Both the MatInspector (http://www.genomatix. de/cgi-bin/eldorado/main.pl) and TFSEARCH (http://www.cbrc.jp/research/db/ TFSEARCH.html) software programs were used to analyze the putative regulatory elements.

For CpG island prediction, CpG Plot (http://www.ebi.ac.uk/Tools/emboss/ cpgplot/) and MethPrimer (http://www.urogene.org/methprimer/indexl.html) software programs were used.

\section{Construction of reporter plasmids for promoter analysis}

Progressive $5^{\prime}$ and $3^{\prime}$ deletion fragments of the POMGNT1 promoter were generated by PCR. Promoter regions containing the mutation were amplified using genomic DNA extracted from patient's total blood sample. As a positive control, a HSV tk-pXP2 construct was generated using a 770-bp fragment of HSV thymidine kinase promoter. All PCR fragments were cloned into the HindIII and XhoI sites of the pXP2 vector (Supplementary Table S1).

\section{Cell culture, transient transfection and reporter gene luciferase assay}

The COS-7 (African Green Monkey kidney fibroblast) and HEK293T (human embryonic kidney) cell lines were maintained at $37^{\circ} \mathrm{C}$ with $5 \% \mathrm{CO}_{2}$ in Dulbecco's modified Eagle's medium (DMEM) supplemented with $10 \%$ fetal bovine serum (both from Gibco, Carlsbad, CA, USA), $100 \mathrm{U} / \mathrm{ml}$ penicillin and $100 \mu \mathrm{g} / \mathrm{ml}$ streptomycin. Approximately $8 \times 10^{5}$ cells were seeded in $60 \mathrm{~mm}^{2}$ culture plates and the cells were then transiently co-transfected with $8 \mu \mathrm{g}$ of the each luciferase reporter construct or the empty vector (negative control) together with $1.6 \mu \mathrm{g}$ of the pSV- $\beta$-galactosidase plasmid as an internal control. The cells were harvested $48 \mathrm{~h}$ after transfection and assayed for both luciferase and $\beta$-galactosidase activities using the Luciferase assay system and the $\beta$-galactosidase enzyme assay system (Promega, Madison, WI, USA). To normalize the variations in transfection efficiency, we divided the measurement of firefly luciferase activity by that of $\beta$-galactosidase activity.

\section{Electrophoretic mobility shift assay (EMSA) and supershift assay} Double-stranded probes containing putative wild-type and mutant binding sites (Supplementary Table S2) were end labelled with $\left[\gamma^{-32} \mathrm{P}\right]$ ATP using T4 polynucleotide kinase (Promega). The nuclear proteins were preincubated on ice for $15 \mathrm{~min}$ in a binding reaction mixture containing $20 \mathrm{~mm}$ Hepes, $70 \mathrm{~mm} \mathrm{NaCl}, 2.5 \mathrm{~mm}$ DTT, $0.05 \%$ Nonidet P-40, $1 \mu \mathrm{g} / \mathrm{ml} \mathrm{BSA,} \%$ Ficoll and
$2 \mu \mathrm{g}$ poly (dI-dC). The ${ }^{32} \mathrm{P}$ end-labelled probe was added and the mixture was incubated for $20 \mathrm{~min}$ at RT. For the competition assay, a 100-fold molar excess of unlabelled probe was added. For the supershift assay, $2 \mu \mathrm{g}$ of Sp1 antibody (sc-420X; Santa Cruz Biotechnology, Inc., Santa Cruz, CA, USA) and control murine IgG were incubated with the nuclear extracts.

\section{Chromatin immunoprecipitation assay (ChIP)}

ChIP assay was carried out as previously described, ${ }^{13}$ using $4 \mu \mathrm{g}$ of the Sp1 antibody (sc-420X) and FLAG M2 antibody (Stratagene, La Jolla, CA, USA). As a negative control, an immunoprecipitation reaction with no antibody was performed. Two different POMGNT1 promoter regions containing the three analyzed Spl-binding motifs were amplified by PCR. A fragment from the coding region of the GAPDH gene was used as a negative control.

\section{Cloning of the cDNA and proximal promoter of the POMGNT1} gene to analyze mRNA and protein expression

The coding region of the hPOMGNT1 gene was amplified using the 3138971 clone from the IMAGE consortium ${ }^{14}$ and cloned into a pcDNA3 vector (Invitrogen, Carlsbad, CA, USA) containing a Flag-tag. This construct, named $\mathrm{P}_{\mathrm{CMV}}$-POMGNT1-pcDNA3-Flag, preserved the CMV promoter of the original vector and served as the positive control. The T7 and CMV promoters of pcDNA3 vector were eliminated and the wild-type and mutant -402 to +644 bp promoter regions were inserted to generate the $\mathrm{P}_{\mathrm{WT}}-P O M G N T 1$ pcDNA3-Flag and $\mathrm{P}_{\mathrm{Mut}}-\mathrm{POMGNT1-pcDNA3-Flag} \mathrm{constructs,} \mathrm{respectively.}$

\section{Western blotting}

COS-7 total protein extracts $\left(30 \mu \mathrm{g}\right.$ of protein from $\mathrm{P}_{\mathrm{CMV}}-$ POMGNT1pcDNA3-Flag transfected cells or $150 \mu \mathrm{g}$ of protein from $\mathrm{P}_{\mathrm{WT} / \mathrm{Mut}^{-}}$ POMGNT1-pcDNA3-Flag transfected cells) were separated on $10 \%$ sodium dodecyl sulphate-polyacrylamide gel electrophoresis (SDS-PAGE) and transferred to a PVDF membrane. The FLAG M2 antibody was used in a dilution of 1:1000. To confirm equal protein loading, the membranes were reprobed with a monoclonal antibody directed against $\beta$-actin (Sigma-Aldrich, St Louis, MO, USA). Signal intensity was quantified by densitometry using Image Gauge software, version 3.3 (Fujifilm, Valhalla, NY, USA).

\section{RT-PCR}

Total RNA was extracted from COS-7 cells using the TRIzol reagent (Invitrogen) according to the manufacturer's instructions. First-strand cDNA was synthesized from $0.5 \mu \mathrm{g}$ of total RNA with $200 \mathrm{U}$ of SuperScript III RNase H reverse transcriptase (Invitrogen). The PCR primers were designed from the Macaca mulatta cDNA sequence of Sp1, Ets1, ZNF202 and GATA-1 to GATA-6 transcription factors (available upon request). A $200 \mathrm{bp}$ fragment of $\beta$-actin was amplified as an internal control for each PCR reaction.

\section{Quantitative RT-PCR}

The assay was performed using the ABI 7900HT Sequence Detection System and Power SYBR Green PCR Master Mix (both from Applied Biosystems, Foster City, CA, USA). For the POMGNT1 gene the primers were: forward, $5^{\prime}$-TGACCC ACTCCCAGACAACA-3'; and reverse, 5'-GTAGCCGTCAATGAAAACTG-3'. The relative expression of the target genes was normalized to that of $18 \mathrm{~S}$ RNA.

\section{Statistical analysis}

Statistical significance was determined using a paired Student's $t$-test and the differences were considered statistically significant when $P<0.05$. Analyses were performed using GraphPad Prism 4.0 software (GraphPad Software, La Jolla, CA, USA).

\section{RESULTS}

\section{Clinical description}

The patient was born after 42 weeks of gestation with a birth weight of $3.49 \mathrm{~kg}$ and a length of $52 \mathrm{~cm}$. Cognitive milestones were acquired at the expected ages, although his initial motor development was slightly delayed. At 9 months of age, the patient could sit without support, but was hypotonic and unable to acquire a sitting position unaided. 
At about 2 years of age, he was unsteady when standing and lacked reflexes to protect himself against falling. Clinical examination revealed muscle weakness, slight generalized amyotrophy and a positive Gower's sign. At the age of 7 years, slight lumbar hyperlordosis was noticed along with difficulties in climbing stairs and continuous fatigue. By 8 years of age, profound weakness of the anterior tibialis muscles and shoulder girdle muscles were observed and his sitting was in a kyphotic position. Ophthalmological examination and a brain MRI revealed normal results. Laboratory evaluation showed a mild elevation of serum creatine kinase ( $283 \mathrm{IU} / \mathrm{l}$ and $389 \mathrm{IU} / 1$ at 2 and 7 years of age, respectively: the normal range is between 36 and $217 \mathrm{IU} / \mathrm{l}$ ).

\section{Histological analysis and immunohistochemistry}

Light microscopy showed pronounced variation in muscle fibre diameters and numerous rounded atrophic fibres (Figure 1a). An increase of the endomysial connective tissue was observed, with fatty infiltration of the perimysium. Muscle fibres undergoing myophagy were occasionally observed and several regenerating basophilic fibres with multiple enlarged nuclei were noted. No large inflammatory infiltrates were observed, nor were larger groups of necrotic and regenerating fibres evident. No abnormal accumulation of neutral lipids was detected. Immunohistochemistry revealed abnormal staining for $\alpha$-DG, with clear immunoreactivity in some fibres and weak or no immunoreactivity in others (Figure 1b), even though normal staining was seen in a control biopsy (Figure 1c). $\beta$-DG, merosin and dystrophin immunoreactivity was normal (data not shown).

\section{Genetic screening}

Genomic DNA screening of the LGMD2O patient revealed a new homozygous mutation in the POMGNT1 promoter, located upstream from the transcription start site (TSS), named -83_-75dup (Figure 1d). This mutation was detected in the heterozygous state in both parents, whereas it was absent in all 200 healthy control individuals.
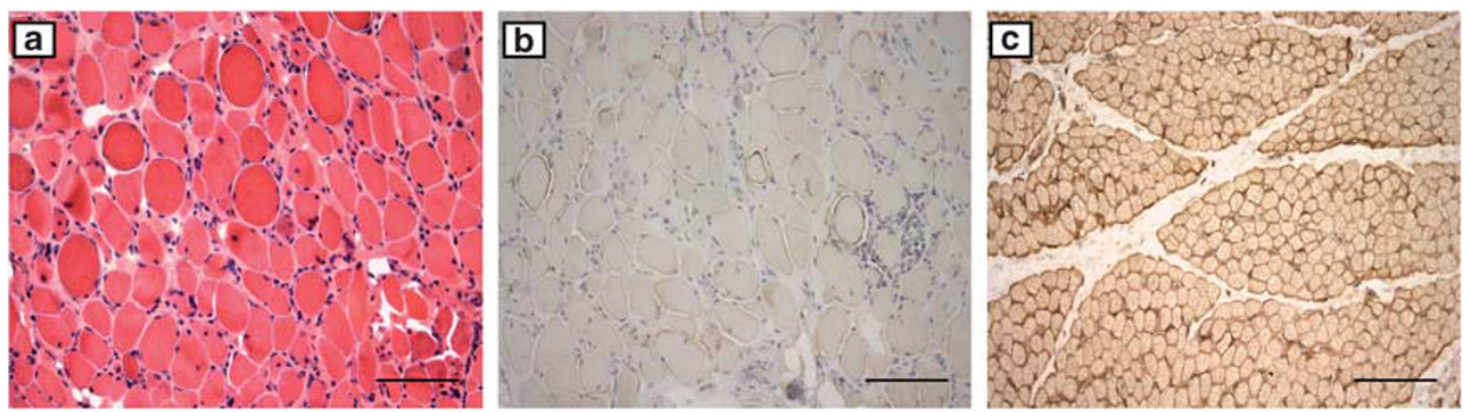

\section{d LGMD2O patient}

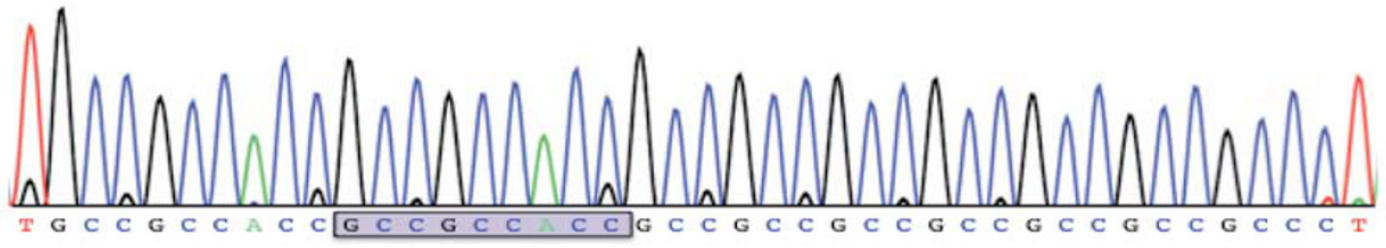

Parents

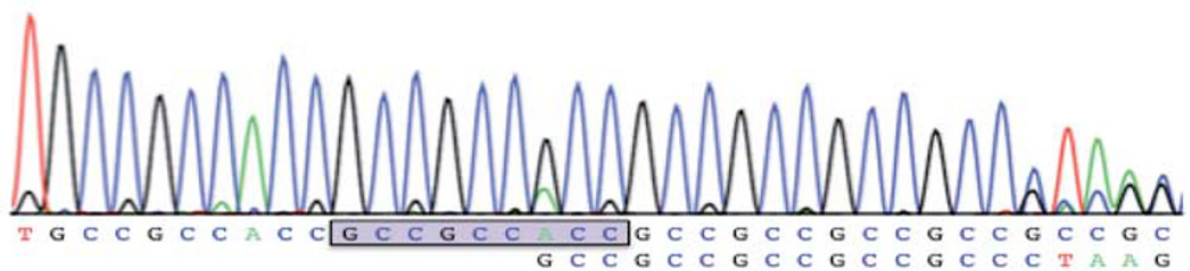

\section{Control}

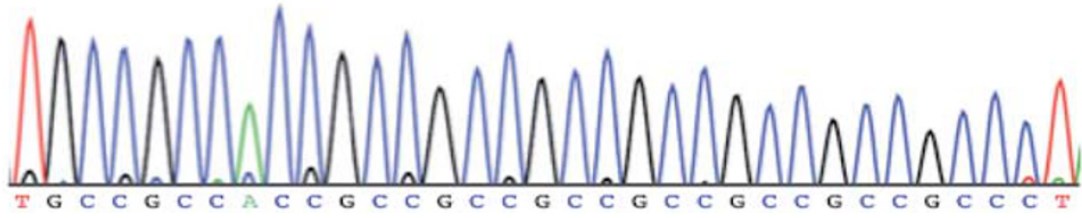

Figure 1 Histopathological findings from the quadriceps muscle biopsy and the sequence analysis of the POMGNT1 gene promoter. (a) Trichrome staining showing variation in fibre size with an increase in endomysial connective tissue and fat in the perimysium. Magnification $\times 20$. (b) Immunolabelling of $\alpha$-DG showing a marked reduction in IIH6 antibody immunoreactivity (avidin-biotin complex method). Magnification $\times 20$. (c) Immunolabelling of $\alpha$-DG in control muscle (avidin-biotin complex method). Magnification $\times 20$, Scale bars $=100 \mu \mathrm{m}$. (d) Electropherograms of the sequence analysis indicating a novel homozygous mutation -83_-75dup (rectangle) in the POMGNT1 promoter region of the LGMD20 patient. Both parents carry the heterozygous form of this mutation. Bottom electropherogram indicates the wild-type sequence. 
Analysis of the $5^{\prime}$-flanking region of the $h P O M G N T 1$ gene

The 2070-bp 5'-flanking region of the hPOMGNT1 gene was examined using the MatInspector and TFSEARCH software programs. This analysis detected several putative transcription factor binding sites, some of which are indicated in Figure 2a. The TSS was set according to the EST sequence BI462048.1, which has an additional 16 nucleotides upstream of the first nucleotide of the published sequence (Gen Bank NM_017739.3). No proximal TATA or CCAAT boxes

a

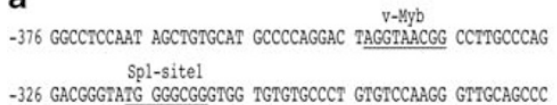

-276 CTCTGACCCT GGCGTGCCCT GTGATCTGGI CCAAGGTGCT AGGCCTCTGA -226 CIGGAGTCGG GCTIOCCAATC GGCGGIGCTG GGAAGCGGCC TGCTCTCGIC

-176 CAGGCAGCCC CACCAGCCAG CCGGGCTGCG AaGCIGCCTA GCACGGICTG -126 AGCAMTCGCC TTACTCGCTG GCTGCTPACA CCCCGACGCC GCIGCCGCCA Spl-site2 spl-site3

-76 CCGCCGCCGC CGCCGCCGCC GCCCTAAGCA CCGCCCGTCG CCCTCTCACC

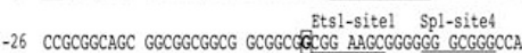
Spl-sites +25 CCGGGGCGGG GCCGCAAGCG GCATGGAGGA GGCGGAGGCC GCGGCGAGCC +75 GGGCCGAGCA GTGAGGGCCC TAGCGGGGCC CGAGCGGGGC CCGGGGCCCC Ets1-site2 +125 TAaCCCGTGA GIGCCTCCGC GTGGGIGTCC GGAACGGGTA GCGCTCCCGG +175 TGCAGGTCIC CIGAGCCCTC GGGCGGCTCC GGGCCCIGGG AGGAGGaGAG Garh-site2 +225 AAGCTGGGGC AGATAGGATG GGACGAGGGI TGAAGACTGG GGCAGGAGAG AML-1a IK-2 +275 GTCGCTCTTT GCTCACCACA GGCTTGGGAA GGCCTGGAAC TTGGTCCTT +325 TAGGGGCTAA $(+335 \ldots \ldots \ldots \ldots+634)$ CCAAICCGG? +644 were found in this region, although the CpGPlot and MethPrimer search programmes identified a CpG island of $440 \mathrm{bp}$ within the region $-198 /+219$ relative to the TSS.

\section{Functional characterization of the wild-type hPOMGNT1} promoter region

To localize the DNA elements critical for promoter activity, we generated a series of bidirectional deletion constructs of the 2070-bp

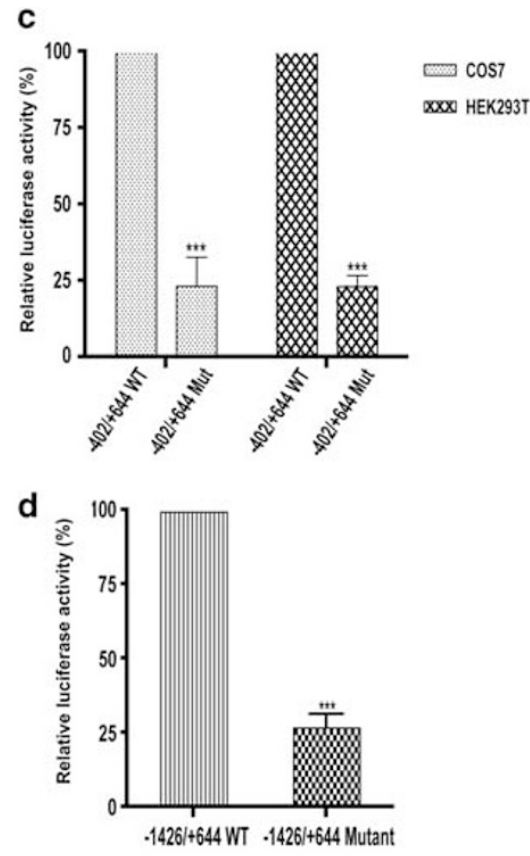

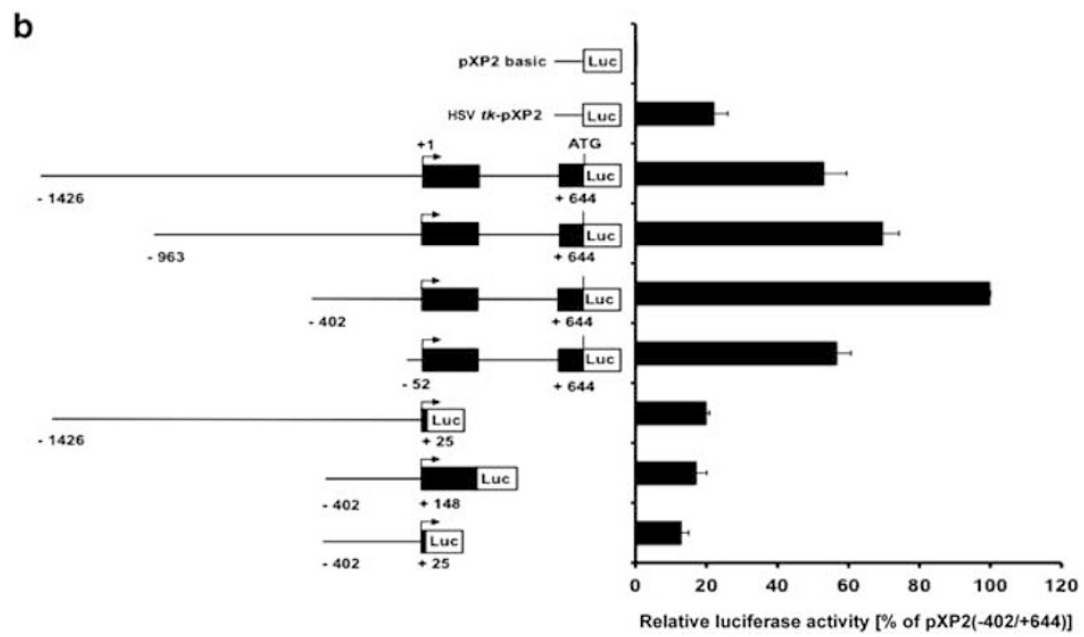

Figure 2 Characterization of the 5'-flanking region of the hPOMGNT1 gene. (a) Nucleotide sequence of the hPOMGNT1 promoter. The putative transcription factor binding sites are underlined and the names annotated. The transcription start site (TSS) is shown as +1 in bold and boxed; the duplicated sequence is shaded. (b) Reporter gene analysis of the 5'-flanking region of the hPOMGNT1 gene. The schematic diagram on the left indicates the hPOMGNT1 promoter luciferase gene constructs analyzed. The fragments of the POMGNT1 promoter mutants were inserted into the pXP2 vector and co-transfected with the pSV$\beta$-galactosidase plasmid into the COS-7 cell line. Normalized Luc activity is shown as a percentage of that of the pXP2(-402/+644) construct. Each bar represents the mean \pm SEM of three independent experiments. (c, d) Reporter gene analysis of the POMGNT1 promoter from the LGMD20 patient. (c) The POMGNT1 promoter region from -402 to +644 bp carrying the homozygous mutation -83 - 75 dup and the wild-type region were cloned into the pXP2 vector. This vector was co-transfected with the pSV- $\beta$-galactosidase plasmid into COS-7 and HEK293T cells. Normalized Luc activity of the -402 to $+644 \mathrm{bp}$ mutant promoter region is shown as a percentage of that of the wild-type pXP2( $-402 /+644)$ plasmid. Each bar represents the mean \pm SEM of three independent experiments. ${ }^{* *} P<0.0001$ (Student's $t$-test). (d) The POMGNT1 promoter region from -1426 to +644 bp carrying the homozygous mutation 83_-75dup and the wild-type region were cloned into the pXP2 vector and co-transfected with the pSV- $\beta$-galactosidase plasmid into the COS-7 cell line. Normalized Luc activity of the -1426 to +644 bp mutant promoter region is shown as a percentage of that of the wild-type pXP2( $-1426 /+644)$ plasmid. Each bar represents the mean \pm SEM of three independent experiments. ${ }^{* *} P<0.0001$ (Student's $t$-test). 
5 -flanking region of the $h P O M G N T 1$ gene. Deletion fragments containing $5^{\prime}$-ends ranging from -1426 to $-52 \mathrm{bp}$ and $3^{\prime}$-ends ranging from +644 to +25 bp were generated by PCR and cloned into the promoterless firefly pXP2 luciferase reporter vector (Figure 2b, left panel). Of all the constructs, the pXP2 $(-402 /+644)$ construct expressed the highest level of promoter activity (Figure $2 \mathrm{~b}$, right panel) and when the 5 -end of this sequence was extended from $-402 \mathrm{bp}$ to $-963 \mathrm{bp}$, there was a slight decrease in luciferase activity. Indeed, extending the $5^{\prime}$ sequence from $-402 \mathrm{bp}$ to $-1426 \mathrm{bp}$ resulted in a $50 \%$ decrease in luciferase activity, suggesting the presence of suppressor activity in this distal 5 '-flanking region. Sequence deletion from $-402 \mathrm{bp}$ to $-52 \mathrm{bp}$ resulted in a reduction in luciferase activity of almost $45 \%$, indicating the presence of a putative regulatory element in this region that is important for promoter activity. Significantly, a binding site for the Sp1 transcription factor was predicted in this region ( $\mathrm{Sp} 1$ site 1$)$.

A significant reduction of nearly $80 \%$ in promoter activity was observed after eliminating the +148 to +644 bp region, which contains a predicted binding site for GATA family transcription factors (GATA site 2). Further deletion of the region +25 to +148 bp produced a decrease of nearly $90 \%$ in the promoter activity of the pXP2 $(-402 /$ $+24)$ and pXP2 $(-1426 /+24)$ constructs.

\section{Mutant hPOMGNT1 promoter significantly inhibits luciferase activity}

We used a luciferase reporter assay to determine whether the -83_-75dup mutation detected in the promoter of the LGMD2O patient's POMGNT1 gene could affect its expression. Transient transfection in COS-7 and HEK293T cells revealed a 75\% decrease in activity of the mutant pXP2(-402/+644Mut) construct when compared with the wild-type construct in both cell lines (Figure 2c). The same result was obtained for the mutant pXP2(-1426/+644Mut) construct (Figure 2d), suggesting that the -83_-75dup mutation strongly affected promoter activity in this patient.

\section{Transcription factor binding to the wild-type hPOMGNT1 proximal promoter}

The deletion analysis suggested that the -402 to +644 bp region represents the proximal promoter of the POMGNT1 gene and that it contains essential transcription factor binding sites required for gene expression. In order to confirm whether Sp1, Ets1 and members of the GATA family of transcription factors bind to the POMGNT1 promoter, EMSA analysis was performed using radiolabelled probes containing: the Sp1 sites 1, 4, and 5; the Ets1 site 1; and the GATA site 2 (Figure 3a). When the wild-type radiolabelled probes were incubated with COS-7 nuclear extracts, a major band was retarded. The specificity of this binding was evident as the formation of the DNAprotein complexes could be inhibited by competition with a 100 -fold molar excess of cold wild-type probe but not with a 100-fold molar excess of the cold mutant probe.

The identity of the Sp1 protein in the DNA-protein complexes was further confirmed in supershift assay, in which the DNA-protein complexes faded away (Figure $3 \mathrm{~b}$ ). ${ }^{15}$ We evaluated the conservation of the three Sp1-binding sites in the Macaca mulatta POMGNT1 promoter (homologous to Chlorocebus aethiops) and multiple alignments using the Clustal W software program (http://www.ebi.ac.uk/ Tools/msa/clustalw2/) revealed a high degree of conservation between the two species for the Sp1-, Ets1- and GATA-binding sites (Figure 3c). To confirm that Sp1 bound to the three EMSA-detected sites in vivo, we performed ChIP assay using chromatin from COS-7 cells (Figure 3d). Two $111 \mathrm{bp}$ and $143 \mathrm{bp}$ fragments of the POMGNT1 promoter were amplified using DNA samples immunoprecipitated with the Sp1 antibody as a template. Together with a $144 \mathrm{bp}$ fragment corresponding to a coding region of GAPDH gene, these two fragments were also amplified using the input material as templates. When DNA samples immunoprecipitated with an anti-FLAG M2 antibody or with no antibody were used as templates, no fragments were amplified with either the POMGNT1 or GAPDH primers.

The transcriptional repressor ZNF202 binds strongly to the mutant proximal promoter of the POMGNT1 gene

Given the decrease in luciferase activity of the LGMD2O patient's POMGNT1 promoter, we reassessed the potential transcription factor binding sites and we identified a putative binding site for the transcriptional repressor ZNF202 in the wild-type promoter region (Figure 2a). The mutation in the LGMD2O patient generated a second binding site for this transcription factor, which suggested that the repression of this gene's transcription might be stronger following the introduction of this mutation. To confirm this in silico-based hypothesis, an EMSA assay was performed to confirm the binding of ZNF202 using the following probes: the wild-type probe containing the putative ZNF202 binding site (ZNF202 WT), the probe containing the mutant ZNF202 site (ZNF202 Mut) and the probe containing the 83_-75dup mutation (ZNF202 Dup) (Supplementary Table S2). No DNA-protein complexes were formed when the nuclear extracts were incubated with the labelled ZNF202 WT probe, whereas a strongly labelled band was retarded when the labelled ZNF202 Dup probe was used (Figure $4 \mathrm{a}$ ). The specificity of this interaction was confirmed by the absence of a band when the labelled ZNF202 Mut probe was used. This labelled band could not be competed by a 100-fold molar excess of cold ZNF202 WT or ZNF202 Mut probes, demonstrating that the wild-type promoter showed no affinity for this transcription factor. However, competition with an excess of cold ZNF202 Dup probe abolished the gel shift because of the strong association of the nuclear extracts with the promoter region containing the mutation. It was not possible to identify ZNF202 as part of the protein complex bound to the mutant proximal promoter by supershift or ChIP assay as no commercial specific antibodies were available. However, ZNF202 was identified in gel shift assay using a control probe containing a previously described ZNF202 consensus sequence (ZNF202 Ctrl: Supplementary Table S2). ${ }^{16}$ Incubation of the radiolabelled ZNF202 Dup probe with a nuclear extract generated a complex band, which was no longer evident when binding was competed by the addition of a 100-fold molar excess of cold ZNF202 Ctrl probe (Figure 4a). We also examined the endogenous expression of Sp1, Ets1, ZNF202 and the GATA-1 to GATA- 6 transcription factors in the COS-7 cell line by RT-PCR, confirming that all the transcription factors analyzed, except for GATA-4, were expressed in COS-7 cells (Supplementary Figure 1).

\section{Transcriptional repression and altered POMGNT1 protein expression in the LGMD2O patient}

Because we had no access to RNA or muscle samples from the patient, we performed an in vitro approach to assess the transcriptional activity of the POMGNT1 promoter. When analyzed by qRT-PCR, the expression of the POMGNT1 gene was by $\sim 50 \%$ in cells transfected with the construct containing the mutant promoter (Figure $4 \mathrm{~b}$ ). Likewise, in western blots, a 70\% decrease in the POMGNT1 protein was detected for the mutant construct when compared with the wild type (Figures $4 \mathrm{c}$ and $\mathrm{d}$ ). Together, these findings suggest that ZNF202 transcription factor strongly suppresses promoter activity, provoking a decrease in the endogenous POMGNT1 mRNA and protein expression. 
a

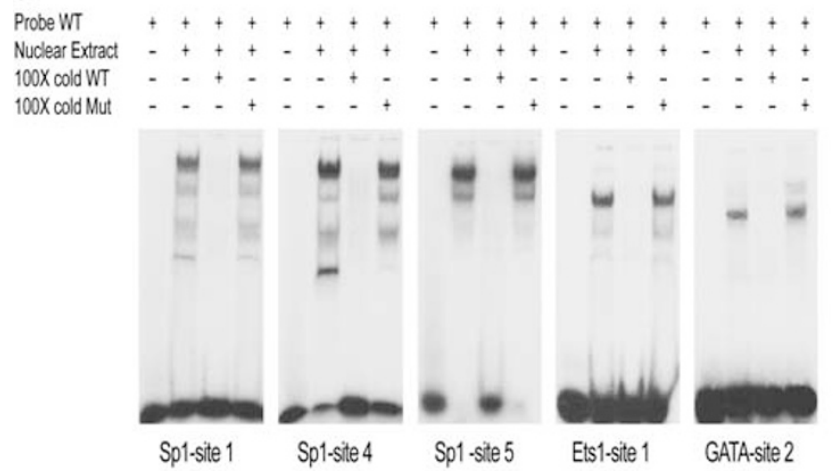

C

Homo sapiens AACGGCCTTGCCCAGGACGGGTATGGGGCGGTGGTGTGTGCCCTGTGTC Macaca mulatta AACGGCCTTGTCCAGGAGGGTATGGGGAGGCTGGTGTGTGTGCTGTGTC

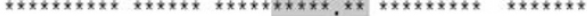

Homo sapiens Macaca mulatta

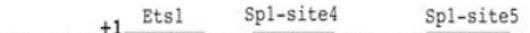
GCGGCGGCGGCGGAAGCGGGGGGCGGGCCAGGCCGGGGCGGGCCGCAA GCGGCGGCGGCGGAAGCGGGGGGGCGGGCCAGGCCGGGGCGGGGCCGCAA $* * * * * * * * * * * * * * * * * * * * * * * * * * * * * * * * * * * * * * * * * * * * * * * * * * * * * *$

GATA

Homo sapiens GGAGGAGGAGAGAAGCTGGGGCAGATAGGATGGGACGAGGGTTGAAGACT Macaca mulatta GGAGGAG---AGAAGCTGGGGTAGATAGGATGGGACGAGGGTTGAAGACT

\section{b}
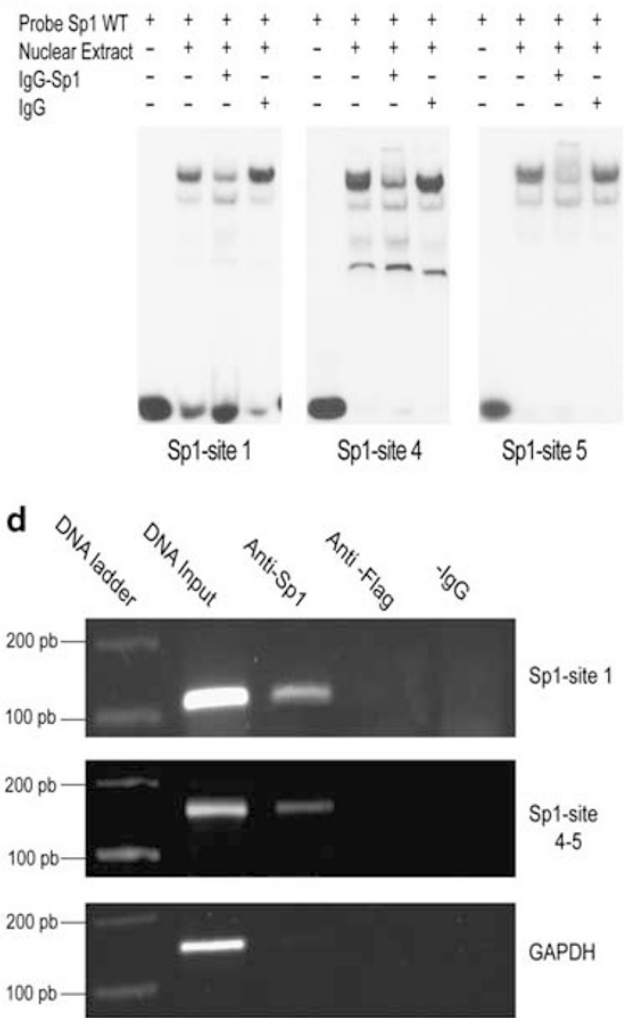

Figure 3 Identification of the transcription factors that bind to the hPOMGNT1 proximal promoter. (a) Electrophoresis mobility shift assay of the POMGNT1 promoter region. The ${ }^{32} \mathrm{P}$-labelled wild-type (WT) probes containing Sp1-binding sites 1,4 and 5 (first three panels, from left to right), the Ets1-binding site 1 (fourth panel) and the GATA-binding site 2 (fifth panel) were incubated with COS-7 nuclear extracts. For each panel: lane 1, free labelled probe (negative control); lane 2, specific DNA-protein complexes whose formation was hampered by co-incubation with an excess of the cold WT probe (lane 3) but not by co-incubation with an excess of cold Mut probe (lane 4). (b) Sp1 supershift assay. For each panel: lane 1, free labelled probe (negative control). ${ }^{32}$ P-labelled probes containing the Sp1-binding sites 1 (first panel), site 4 (second panel) and site 5 (third panel) were incubated with COS-7 nuclear extracts. Lane 2, specific DNA-protein complexes whose formation was prevented by the addition of anti-Sp1 antibody (lane 3) but not by a mouse IgG (lane 4). (c) Sequence comparison of Sp1-, Ets1- and GATA-binding sites between Homo sapiens and Macaca mulatta (Gen Bank accession no. NC_007858.1). The highly conserved transcription factor binding sites are shaded. (d) Chromatin immunoprecipitation assay was performed on the COS-7 cell line. Proteins were crosslinked to the DNA with formaldehyde, and the chromatin was subsequently sonicated and pre-cleared with protein G beads. Lane 1, DNA ladder; lane 2, DNA input. Equivalent amounts of chromatin were immunoprecipitated by antibodies against Sp1 (lane 3) or FLAG (nonspecific antibody used as negative control; lane 4). Lane 5, sample without antibody. PCR was performed using the POMGNT1 (top and middle panels) and GAPDH primers (bottom panel).

\section{DISCUSSION}

The POMGNT1 gene is located on chromosome 1p34.1 and it encodes a 660-amino acid type II transmembrane protein that catalyzes the formation of GlcNAc $\beta 1-2$ Man linkage of O-mannosyl glycans. ${ }^{5}$ Mutations in POMGNT1 were almost exclusively associated with MEB, although a LGMD patient with severe myopia and normal intellect was reported. ${ }^{9}$ This LGMD patient carried a novel homozygous missense mutation in the coding region of POMGNT1 and displayed a subtle reduction in $\alpha$-DG immunolabelling in skeletal muscle.

Here we describe a second LGMD patient without brain abnormalities, mental retardation or ocular involvement, and who carries a homozygous 9-bp duplication (-83_-75dup) in the promoter region of the POMGNT1 gene. The changes observed in the muscle biopsy from this patient were compatible with a muscular dystrophy, and extensive immunohistochemical analysis suggested a diagnosis of $\alpha$-dystroglycanopathy: a mosaic pattern of IIH6-positive and IIH6negative fibres across the muscle as previously described in some LGMD2I patients, ${ }^{17}$ including one CMD-MR patient who carried two heterozygous mutations in the POMT1 gene. ${ }^{18}$ Labelling of other membrane proteins was positive, precluding other types of LGMD. Thus, this individual is the first dystroglycanopathy patient to be described carrying a mutation in the promoter region of one of the genes directly or indirectly implicated in the $\alpha$-DG glycosylation pathway.

Gene reporter analysis showed that the -402 to +644 bp region of the POMGNT1 promoter exhibited maximal luciferase activity. In turn, the same region carrying the $9 \mathrm{bp}$ duplication found in the LGMD2O patient showed a 75\% decrease of luciferase activity in two different cell lines, confirming that downregulation of gene expression was not cell line specific. Indeed, similar findings were obtained for the mutant -1426 to +644 bp promoter region. EMSA, supershift and ChIP assays confirmed the existence of three binding sites for the Sp1 transcription factor. These sites contained the $\mathrm{Sp} 1$ consensus-binding sequence (5'-GGGCGG-3') and they were located within the CpG island at the positions: -318 to $-310 \mathrm{bp},+13$ to $+19 \mathrm{bp}$ and +28 to $+34 \mathrm{bp}$. Sp1 belongs to the family of transcription factors with a zincfinger-type DNA binding domain that binds to GC-rich sequences. ${ }^{19}$ In many TATA-less promoters, $\mathrm{Sp} 1$ binding is required to determine the transcription start site. ${ }^{20}$ Another transcription factor potentially 


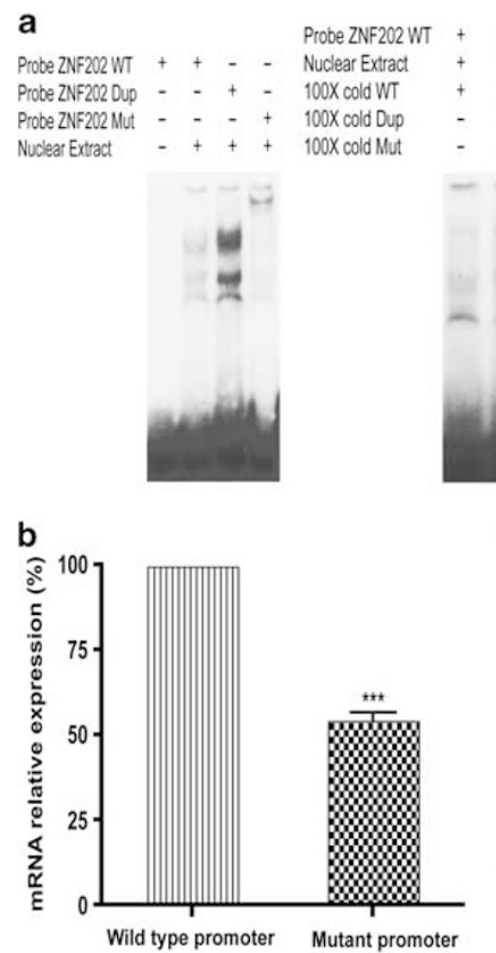

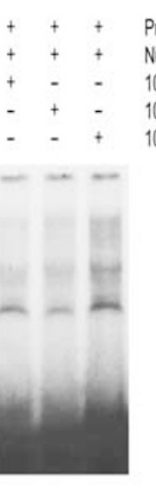
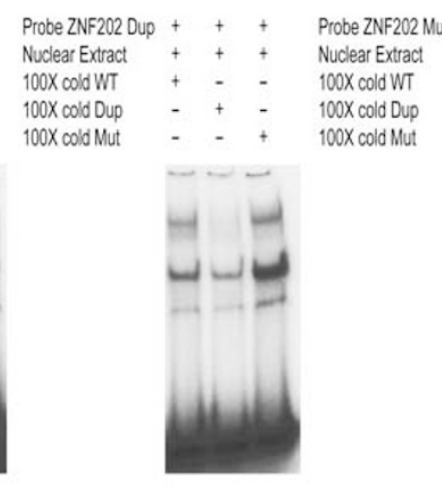

C

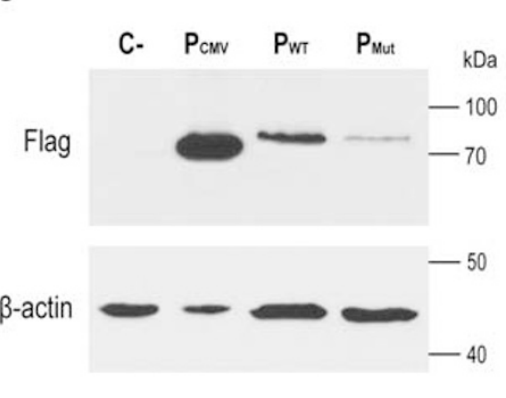

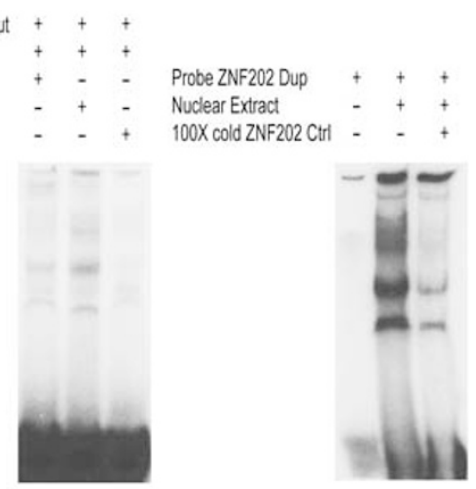

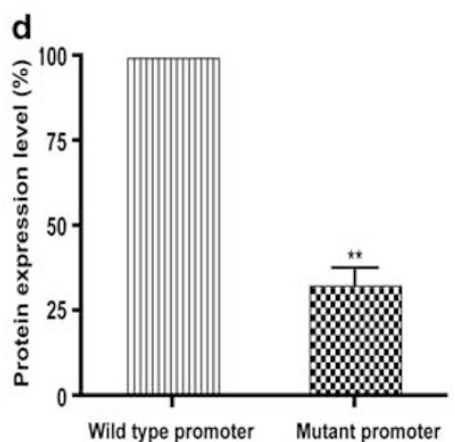

Figure 4 Functional characterization of the POMGNT1 promoter mutation -83_-75dup. (a) Electrophoresis mobility shift assay shows that ZNF202 transcription factor binds to the mutant POMGNT1 promoter. First panel: lane 1, free labelled probe (negative control). COS-7 nuclear extracts bound to labelled ZNF202 Dup probe (lane 3) but not to ZNF202 WT probe (lane 2) and ZNF202 Mut probe (lane 4). Competition of the ${ }^{32}$ P-labelled ZNF202 WT probe (second panel), ${ }^{32}$ P-labelled ZNF202 Dup probe (third panel) and ${ }^{32}$ P-labelled ZNF202 Mut probe (fourth panel) using an excess of the cold probes. Fifth panel: lane 1, free-labelled probe (negative control); lane 2, specific DNA-protein complexes whose formation was impaired by the addition of an excess of cold ZNF202 Ctrl probe (lane 3). (b-d) Decreased expression of POMGNT1 mRNA and protein in the LGMD2O patient. The three constructs together with the empty vector (C-) were transfected into COS-7 cells. Total RNA and protein was extracted $48 \mathrm{~h}$ later. (b) POMGNT1 mRNA expression detected by qRTPCR. The mutant promoter activity was normalized to $18 \mathrm{~S}$ RNA and expressed relative to that of the wild-type promoter. Each bar represents the mean \pm SEM of three independent experiments. ${ }^{* *} P<0.0001$ (Student's $t$-test). (c) The amount of POMGNT1 (top panel) and actin (bottom panel) protein was determined by western blotting assays. The CMV promoter (lane 2), wild-type (lane 3) or mutant (lane 4) POMGNT1 promoters drove gene expression. Individual western blots were performed three times and a representative image is shown. (d) Quantification of POMGNT1 protein normalized to actin. POMGNT1 protein produced by the mutant promoter was expressed relative to that of wild type. Each bar represents the mean \pm SEM of three independent experiments. ${ }^{*} P<0.009$ (Student's $t$-test).

implicated in the transcriptional regulation of POMGNT1 gene is Ets1, which binds to the core (5'-GGAA/T-3') sequence. Our EMSA assay detected one Ets1-binding site located in the +2 to +8 bp region. Sp1/ Sp3, AP1 and SRF are thought to be coregulators of Ets1 binding. ${ }^{21}$ The +233 to +241 bp promoter region contains a GATA-binding motif that exhibits the nucleotide sequence $5^{\prime}$-(A/T)GATA(A/T)-3'. RT-PCR assays demonstrate that COS-7 cells endogenously express all members of the GATA transcription factor family, except GATA-4.

The experiments described here represent the first functional characterization of the POMGNT1 gene and they demonstrate the importance of the Sp1, Ets1 and GATA transcription factors for the transcriptional activity of this gene. The promoter regions of the other putative or demonstrated glycosyltransferases involved in the glycosylation pathway of $\alpha$-DG also contain potential binding sites for these transcription factors, which may account for a common transcriptional regulatory mechanism (data not shown). The complete functional analysis of the POMGNT1 gene promoter harbouring the -83_-75 dup mutation revealed a reduction in gene expression, demonstrated by the low levels of POMGNT1 mRNA and protein. This decrease in promoter activity may be associated with the strong binding of the transcription factor ZNF202 to the mutant promoter, which contains two consecutive ZNF202 binding sites. Interestingly, the wild-type promoter contains a single predicted ZNF202-binding site but this does not appear to be sufficient to bind to ZNF202 in vitro. The influence of this transcription factor on POMGNT1 expression is supported by the strong in vitro binding of the transcriptional repressor to direct repeats of the consensus binding motif $\left(5^{\prime}\right.$ GGGGT-3') in the promoter region of several genes involved in lipid metabolism, such as apolipoprotein AIV, apolipoprotein CIII and lipoprotein lipase, as well as to genes involved in blood vessel maintenance like VEGF. ${ }^{16}$ In addition to its affinity for GnT motifs, ZNF202 can bind to GC boxes, and in our case, ZNF202 bound to direct repeats of the $5^{\prime}$-GCCA-3' motif. Structurally, ZNF202 contains a SCAN (SRE-ZBP, CT-finS1, AW-1 and Number 18) oligomerization domain, a KRAB (Krüppel-associated box) repression domain and eight zinc-finger DNA-binding motifs. For TATA box-containing promoters the mechanism of ZNF202-induced transcriptional repression may be mediated by the KRAB repression domain, which interferes with the TATA box-dependent basal transcription machinery. ${ }^{22}$ Further studies will be necessary to elucidate the mechanism by which ZNF202 mediates transcriptional repression for promoters lacking a TATA box.

To date, in dystroglycanopathy patients defects have been described in six putative or demonstrated glycosyltransferases (POMT1, 
POMT2, POMGNT1, LARGE, FKTN and FKRP), two accessory proteins of glycosyltransferases (DPM3 and DPM2) and the dystroglycan protein. ${ }^{1}$ Despite this genetic variability, the mutations reported account for only about $50 \%$ of dystroglycanopathy patients. Moreover, to our knowledge, no alterations in the promoter regions of these genes have been reported previously. Thus, we describe here the first alteration in the promoter of a gene involved in the $\alpha$-DG glycosylation pathway, which diminishes the POMGNT1 mRNA and protein levels because of ZNF202-mediated repression of gene transcription. Our data underline the importance of screening the promoter and other $5^{\prime}$-upstream regulatory regions in dystroglycanopathy patients in whom mutations are not detected in the gene-coding region. Mutations within regulatory elements or transcription factor binding sites can affect normal transcriptional activity, resulting in the synthesis of hypoglycosylated $\alpha$-DG. Therefore, the complete functional characterization of the promoters of previously described genes could help identify the genetic bases for the many dystroglycanopathies of currently unknown aetiology.

\section{CONFLICT OF INTEREST}

The authors declare no conflict of interest.

\section{ACKNOWLEDGEMENTS}

We thank Cristina Lendínez and Belén García for their excellent technical assistance, and Mark Sefton for critical reading of the manuscript. This work was supported by a grant from the Fondo de Investigaciones Sanitarias (PI09/0343). MR is supported by a FPU-UAM fellowship. JB is supported by a fellowship of the Fund for Scientific Research (FWO-Flanders).

1 Godfrey C, Foley AR, Clement E, Muntoni F: Dystroglycanopathies: coming into focus. Curr Opin Genet Dev 2011; 21: 278-285.

2 Hewitt JE: Abnormal glycosylation of dystroglycan in human genetic disease. Biochim Biophys Acta 2009; 1792: 853-861.

3 Campbell KP: Three muscular dystrophies: loss of cytoskeleton-extracellular matrix linkage. Cell 1995; 80: 675-679.
4 Manya H, Chiba A, Yoshida A et al: Demonstration of mammalian protein O-mannosyltransferase activity: coexpression of POMT1 and POMT2 required for enzymatic activity. Proc Natl Acad Sci USA 2004; 101: 500-505.

5 Yoshida A, Kobayashi K, Manya $\mathrm{H}$ et al: Muscular dystrophy and neuronal migration disorder caused by mutations in a glycosyltransferase, POMGnT1. Dev Cell 2001; 1: 717-724.

6 Muntoni F, Torelli S, Brockington M: Muscular dystrophies due to glycosylation defects. Neurotherapeutics 2008; 5: 627-632.

7 Manya H, Sakai K, Kobayashi K et al: Loss-of-function of an N-acetylglucosaminyltransferase, POMGnT1, in muscle-eye-brain disease. Biochem Biophys Res Commun 2003; 306: 93-97.

8 Taniguchi K, Kobayashi K, Saito $\mathrm{K}$ et al: Worldwide distribution and broader clinical spectrum of muscle-eye-brain disease. Hum Mol Genet 2003; 12: 527-534.

9 Clement EM, Godfrey C, Tan J et al: Mild POMGnT1 mutations underlie a novel limbgirdle muscular dystrophy variant. Arch Neurol 2008; 65: 137-141.

10 Guglieri M, Straub V, Bushby K, Lochmuller H: Limb-girdle muscular dystrophies. Curr Opin Neurol 2008; 21: 576-584.

11 Martin JJ, Ceuterick C, Van Goethem G: On a dominantly inherited myopathy with tubular aggregates. Neuromuscul Disord 1997; 7: 512-520.

12 Nordeen SK: Luciferase reporter gene vectors for analysis of promoters and enhancers. Biotechniques 1988; 6: 454-458.

13 Lou G, Li Y, Chen B et al: Functional analysis on the $5^{\prime}$-flanking region of human FXR gene in HepG2 cells. Gene 2007; 396: 358-368.

14 Lennon G, Auffray C, Polymeropoulos M, Soares MB: The I.M.A.G.E. Consortium: an integrated molecular analysis of genomes and their expression. Genomics 1996; 33: 151-152.

15 Won J, Yim J, Kim TK: Sp1 and Sp3 recruit histone deacetylase to repress transcription of human telomerase reverse transcriptase (hTERT) promoter in normal human somatic cells. J Biol Chem 2002; 277: 38230-38238.

16 Wagner S, Hess MA, Ormonde-Hanson P et al: A broad role for the zinc finger protein ZNF202 in human lipid metabolism. J Biol Chem 2000; 275: 15685-15690.

17 Brown SC, Torelli S, Brockington $\mathrm{M}$ et al: Abnormalities in alpha-dystroglycan expression in MDC1C and LGMD2I muscular dystrophies. Am J Pathol 2004; 164: 727-737.

18 Jimenez-Mallebrera C, Torelli S, Feng L et al: A comparative study of alpha-dystroglycan glycosylation in dystroglycanopathies suggests that the hypoglycosylation of alphadystroglycan does not consistently correlate with clinical severity. Brain Pathol 2009; 19: 596-611.

19 Smale ST, Schmidt MC, Berk AJ, Baltimore D: Transcriptional activation by Sp1 as directed through TATA or initiator: specific requirement for mammalian transcription factor IID. Proc Natl Acad Sci USA 1990; 87: 4509-4513.

20 Jolliff K, Li Y, Johnson LF: Multiple protein-DNA interactions in the TATAA-less mouse thymidylate synthase promoter. Nucleic Acids Res 1991; 19: 2267-2274.

21 Sharrocks AD: The ETS-domain transcription factor family. Nat Rev Mol Cell Biol 2001; 2: 827-837.

22 Pengue G, Lania L: Kruppel-associated box-mediated repression of RNA polymerase II promoters is influenced by the arrangement of basal promoter elements. Proc Natl Acad Sci USA 1996; 93: 1015-1020.

Supplementary Information accompanies the paper on European Journal of Human Genetics website (http://www.nature.com/ejhg) 